\title{
Isolation of High Level Macrolide Resistant Bordetella pertussis Without Transition Mutation at Domain V in Iran
}

\author{
Bahman Mirzaei ${ }^{1}$; Zakaria Bameri ${ }^{1}$; Ryhane Babaei ${ }^{1}$; Fereshteh Shahcheraghi ${ }^{1, *}$ \\ ${ }^{1}$ Department of Bacteriology, Pasteur Institute of Iran, Tehran, IR Iran \\ *Corresponding author: Fereshteh Shahcheraghi, Department of Bacteriology, Pasteur Institute of Iran, Tehran, IR Iran. Tel: +98-2166953311, Fax: +98-2166953331, \\ E-mail: shahcheraghifereshteh@yahoo.com
}

Received: February 15, 2014; Revised: July 31, 2014; Accepted: September 24, 2014

\begin{abstract}
Background: Bordetella pertussis, as a causative agent of whooping cough, due to the annual rise y of infection cases, failure of prophylaxis and treatment by macrolides, is considered as the new concern in the health care system.

Objectives: The main objective of this study was the determination of single nucleotide polymorphisms (SNPs) at domain V, as the main binding site for macrolides, following the identification of high level macrolides resistant $B$. pertussis.

Materials and Methods: Following the identification of 11 recovered B. pertussis isolates, from a total of 1084 nasopharyngeal swabs, by using the biochemical and molecular methods, the activities of erythromycin, azithromycin and clarithromycin antibiotics against the recovered isolates were examined. Subsequently, A-G transition mutations in domain V were analyzed by molecular techniques, such as Polymerase Chain Reaction-Restriction Fragment Length Polymorphism (PCR-RFLP) and sequencing.

Results: After susceptibility testing, one strain was detected as a high level macrolide resistant B. pertussis (Erythromycin $=128 \mu \mathrm{g} / \mathrm{mL}$, Clarithromycin $>256 \mu \mathrm{g} / \mathrm{mL}$ ). After sequencing and PCR-RFLP methods, transition mutations in positions 2047 and 2058 of the mentioned domain were not observed.

Conclusions: Although previous studies have shown that A-G transition mutations in 23 SrRNA gene (domain V) are the main reason for the occurrence of high level macrolides resistance in B. pertussis, however, the mentioned single nucleotide polymorphisms (SNPs) have not been detected in our resistant strain. This is the first report of high level macrolide resistant B. pertussis, without SNPs in domain V, in Iran.
\end{abstract}

Keywords: Drug Resistance, Minimum Inhibitory Concentration; Macrolides; Bordetella pertussis; Mutation

\section{Background}

Whooping cough (pertussis) is the acute respiratory infectious disease caused by the Bordetella pertussis bacterium (1). The B. pertussis, as a causative agent of pertussis, is transferred through droplets produced by coughing, sneezing, or talking (2). Infants are the age group with the highest risk of morbidity, hospitalization and mortality. Estimates from World Health Organization (WHO) suggest that, in 2008, about 16 million cases of pertussis occurred worldwide, 95\% of which were in developing countries, and that about 195000 children died from the disease (3). Despite the vaccination, the incidence of pertussis in the vaccinated individuals is rising. Although macrolides are used as the drug of choice for prevention and treatment of pertussis, considering previous reports for the isolation of high level macrolides resistant strains, the emergence of resistant strains must be regarded as an alert signal for the health care system $(1,4-6)$.

Antimicrobial drug treatment substantially contributes to the control of disease transmission (6). According to the published study by Weisblum, in 1995, peptidyle transferase activity is a main characteristic of macrolide binding site of domain $\mathrm{V}(7)$. Following detection of first erythromycin resistant $B$. pertussis in Arizona, four additional cases were detected in USA(8). Recently, two strains of erythromycin resistant $B$. pertussis were reported in France and China, too $(5,6)$. Transition mutations (A-G) in nucleotide positions 2047 and 2058 (critical erythromycin binding site in 23SrRNA gene) have been demonstrated, as a main cause of high level resistance against macrolides in B. pertussis (4-6).

\section{Objectives}

The main objective of this study is to determine the single nucleotide polymorphisms (SNPs) at domain V, as main binding site for macrolides, following the identification of high level macrolides resistant B. pertussis.

\section{Materials and Methods}

From May 2009 to December 2010, 1084 nasopharyngeal swab specimens from the patients having pertussis symptoms, with the age ranging from $<1$ month to $>90$

Copyright (C) 2015, Ahvaz Jundishapur University of Medical Sciences. This is an open-access article distributed under the terms of the Creative Commons Attribution-NonCommercial 4.0 International License (http://creativecommons.org/licenses/by-nc/4.0/) which permits copy and redistribute the material just in noncommercial usages, provided the original work is properly cited. 
years, were delivered to the Pertussis Reference Laboratory at the Pasteur Institute of Iran, Tehran, Iran. Specimen swabs (Dacron-tipped swabs) were transported from the different provinces of Iran to the laboratory, on ReganLowe transport medium (Difco, Becton Dickinson, Franklin Lakes, NJ, USA).

\subsection{Isolation and Identification of Bordetella per- tussis}

Isolation and identification of B. pertussis strains were performed according to the WHO recipes (9). After performing conventional biochemical tests, growth of colonies was confirmed using Taq Man real timepolymerase chain reaction (RT-PCR) assay. The RT-PCR assay was accomplished according to the previously described method (10).

\subsection{Taq Man Real Time Polymerase Chain Reaction}

Taq Man RT-PCR assay was applied for precise determination of $B$. pertussis in clinical samples. Insertion sequence 481 (IS 481) and BP 283 sequence (Codon gene between tyolase region) were targeted by using specific primers and probes $(10,11)$. Utilized primers, probes sequences and amplification protocol in Taq Man RT-PCR assay were described previously $(10,11)$. Taq Man RT-PCR was performed in an ABI 7500 instrument (Applied Biosystems, Waltham, MA, USA). Bordetella pertussis ATCC 9797 served as the control strain.

The amplification plots and standard curve for RT-PCR have been shown in Figure 1.

\subsection{Macrolides Susceptibility Test}

Susceptibility testing was performed according to the Clinical Laboratory Standard Institute (CLSI) 2010 guideline, applying antimicrobial agents, such as erythromycin, azithromycin and clarithromycin (Difco, Becton Dickinson, Franklin Lakes, NJ, USA) on the Muller Hinton Agar (Difco, Becton Dickinson, Franklin Lakes, NJ, USA) containing $5 \%$ sheep blood (12). Minimum inhibitory concentrations (MICs) breakpoints for the mentioned antibiotics were determined according to the recommended guideline in CLSI (12). The concentration of antibiotic agents was prepared between $0.016-512 \mu \mathrm{g} / \mathrm{mL}$ of antibiotics. Antibiotic free plates were inoculated to check for growth and purity. Bordetella pertussis ATCC 9797 and Staphylococcus aureus ATCC 29213 served as control strains. Strains for which the MICs of macrolides were $\leq$ $0.12 \mu \mathrm{g} / \mathrm{mL}$ were considered susceptible $(8,12)$.

\subsection{Pulsed Field Gel Electrophoresis}

Pulsed field gel electrophoresis (PFGE) analysis was done according to the standardized recommendations for epidemiological typing of B. pertussis, in a previously published study (13). Chromosomal DNA samples from 11 strains were digested with XbaI (Fermentas, Vilnius, Lith- uania) and examined by PFGE. Subsequently, the profiles of PFGE were analyzed by using BioNumerics software, version 4.61 (Applied Maths NV, Sint-Martens-Latem, Belgium) (Figure 2). Salmonella Braenderup H9812 was used as molecular weight marker (13).

Figure 1. Taq Man Real Time-Polymerase Chain Reaction Amplification Plots

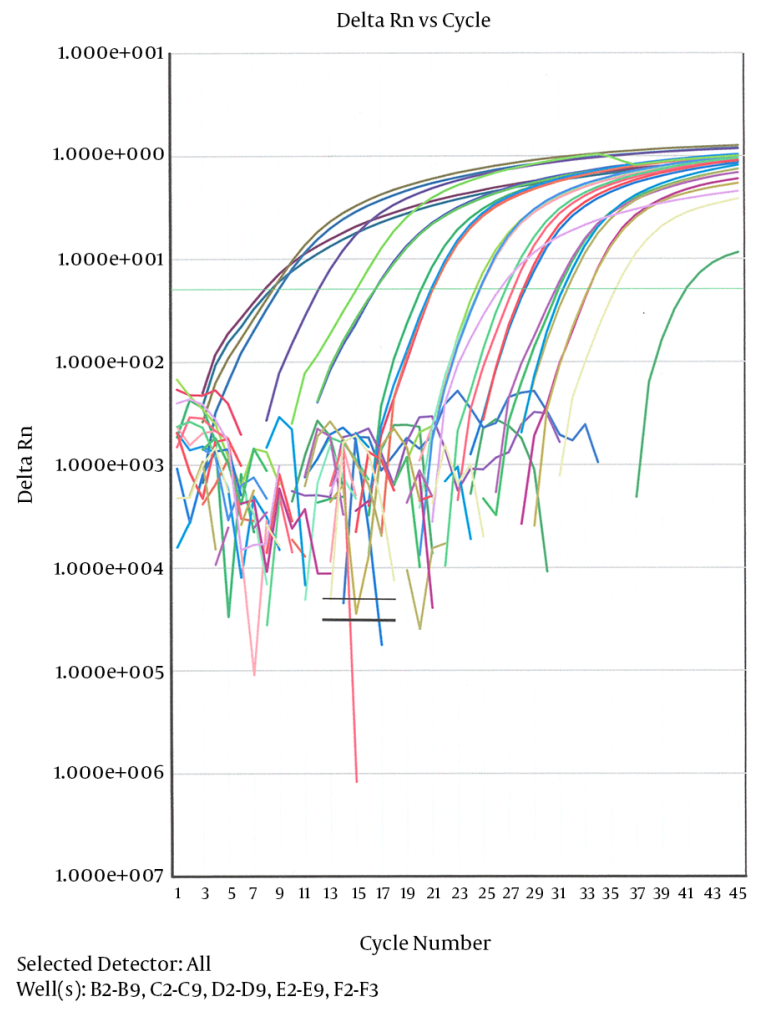

All samples that were positive in culture test were also positive in RT-PCR. They are shown as sharp curve. (Several B. pertussis samples double tested in RT-PCR). Abbreviations: RT-PCR, real time-polymerase chain reaction.

Figure 2. Pulsed Field Gel Electrophoresis Pattern of the Bordetella pertussis Strains Digested With Xbal

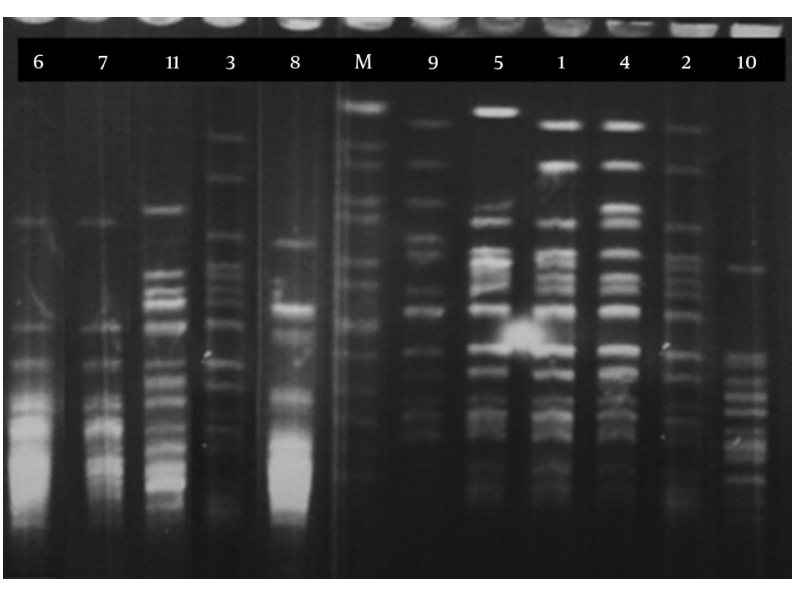

Lane M, genomic pattern of Salmonella Braenderup H9812 as a marker 
Mirzaei B et al.

Figure 3. Amplified 521bp Domain V in Bordetella pertussis Strains by Polymerase Chain Reaction

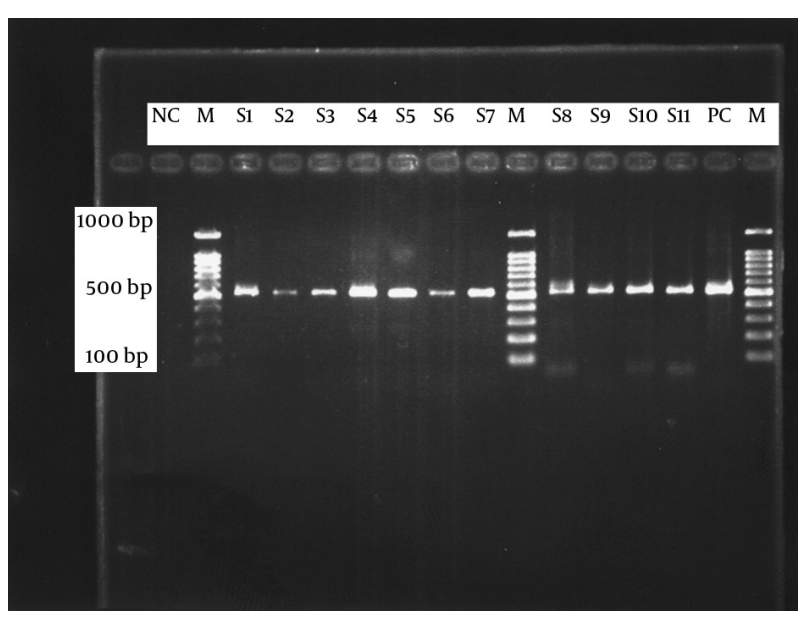

Abbreviations: MP, 100bp marker plus; NC, negative control; PC, positive control; S, samples.

Figure 4. Screening for the A2047G and A2058G Mutations by Polymerase Chain Reaction - Restriction Fragment Length Polymorphism Analysis

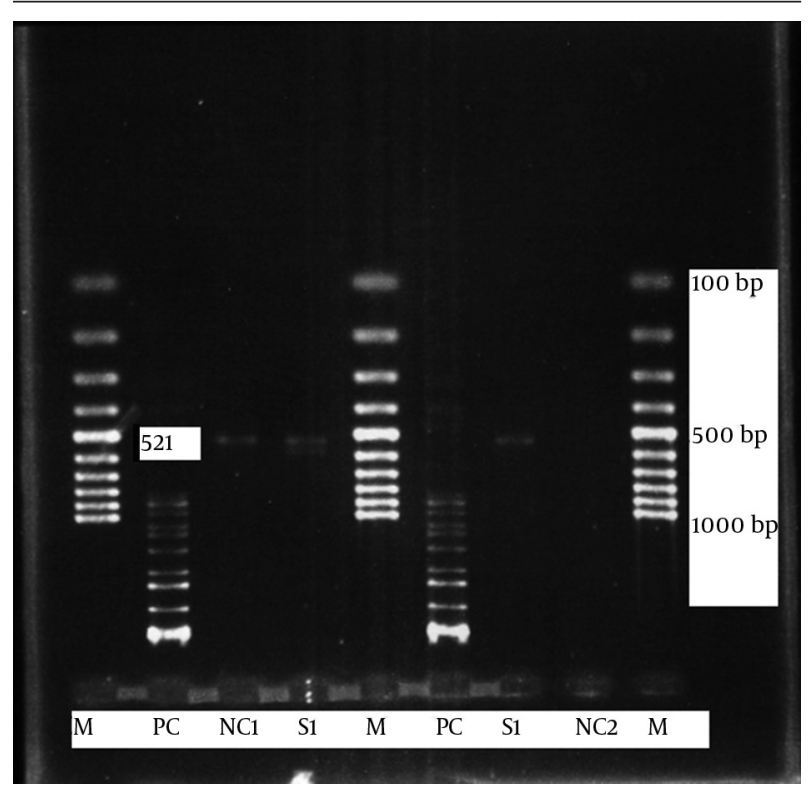

The 521bp fragment of the 23SrRNA gene amplified by PCR from the B. pertussis clinical isolates and controls; Abbreviations: B. pertussis, Bordetella pertussis; PCR, polymerase chain reaction.

\subsection{DNA Extraction}

Genomic DNA was extracted from isolated bacteria by a commercial high pure extraction kit (Roche Diagnostics GmbH, Mannheim, Germany). Quantity of extracted DNA was measured by NanoDrop ND-1000 ${ }^{\circledR}$ Spectrophotometer (NanoDrop, Wilmington, DE, USA) and adjusted to $500 \mathrm{ng} \mu \mathrm{L}^{-1}$.

\subsection{Polymerase Chain Reaction}

The A-G transition mutations at 23SrRNA (domain V) were analyzed by using the PCR method. The amplification protocol was originally described in another study (4). Briefly, PCR was performed by $5 \mu \mathrm{L}$ genomic DNA, as template, in a total volume of $25 \mu \mathrm{L}$, containing $10 \mu \mathrm{L}$ master amplicon (Promega, Fitchburg, WI, USA), 1907U primer 5'/-TTCCTTGTCGGGTAAGTTCC-3'/1pmol/ $\mu \mathrm{L}, 2408 \mathrm{~L}$ primer (Bioneer, Daejeon, Korea) 5'/-GCGGTATCAGCCTGTTATCC-3' $/ 1 \mathrm{pmol} / \mu \mathrm{L}$ and $8 \mu \mathrm{L}$ distilled water. The PCR process was optimized with purified DNA of B. pertussis (ATCC 9797), as positive control. Amplification of 521bp products was performed on Gene Amp PCR system (Applied Biosystems, Waltham, MA, USA) utilizing a program as follows: an initial cycle of denaturation $95^{\circ} \mathrm{C}$ for $5 \mathrm{~min}$ utes, followed by 30 cycles of $95^{\circ} \mathrm{C}$ for 1 minute, $60^{\circ} \mathrm{C}$ for 1 minute and $72^{\circ} \mathrm{C} 1$ minute. Amplified products were visualized on $1.5 \%$ agarose gel (KBC, Maw Pure agarose, Spain) stained by ethidium bromide, by a Gel Logic 212 Pro imaging system (University of Alaska, AK, USA) (Figure 3). Utilized primers were synthesized by Bioneer Corporation (Bioneer, Daejeon, Korea).

\subsection{Sequencing}

The PCR products were purified using a commercial gel purification kit (QIAquick PCR Purification Kit, Qiagen, Venlo, The Netherlands). The sequencing process was accomplished by applying the dye terminal method in an ABI 377 sequencer (Applied Biosystems, Waltham, MA, USA). Sequencing results were interpreted through a query to the Gen Bank local alignment search tool (BLAST) (blast.ncbi.nlm.nih.gov/Blast.cgi?).

\subsection{Restriction Fragment Length Polymorphism}

Transition mutation in domain $\mathrm{V}(23 \mathrm{SrRNA})$ at position 2047 or 2058 is predicted to generate a new restriction site for BbsI enzyme (Fermentase, Vilnius, Lithuania). Amplified products were targeted for the activities of digestive enzymes BbsI, according to the manufacturer recommendations. Finally, products were resolved by Tris Borate EDTA gel by electrophoresis on the $2 \%$ agarose. Restriction fragments length polymorphism (RFLP) pattern was stained and observed in $2 \%$ agarose gel (KBC, Maw Pure agarose, Spain) by a Gel Logic 212 Pro (University of Alaska, AK, USA) instrument (Figure 4).

\section{Results}

Out of 1084 collected nasopharyngeal swabs from suspected patients, 11 specimens (1.01\%) were positive by conventional test, such as bacterial culture. Using the Taq Man RT- PCR, all B. pertussis strains were approved and then, by the PFGE method, genetic relationships between the isolates were determined. To find genetic relationships between the isolates, we obtained genomic patterns of the isolates by PFGE analysis, which revealed six PFGE profiles 
(A-F), among which three and two isolates have the same patterns in profiles A and B, respectively. Hopefully, macrolide resistant $B$. pertussis strain was not among the isolates in profile A, which was considered as a predominant profile (with five isolates) in this research.

Susceptibility tests for mentioned antimicrobial agents showed that: all B. pertussis isolates were susceptible to azithromycin; one isolate of B. pertussis with MIC break point consisting: erythromycin $=128 \mu \mathrm{g} / \mathrm{mL}$, clarithromycin $>256 \mu \mathrm{g} / \mathrm{mL}$, was screened as high level macrolide resistant. Excluding one isolate with MIC $=0.12 \mu \mathrm{g} / \mathrm{mL}$ to azithromycin, all the isolates showed MIC $\leq 0.06 \mu \mathrm{g} / \mathrm{ml}$ to this antibiotic. From 11 strains, which were examined for MIC, strains five and six showed MIC $\geq 2 \mu \mathrm{g} / \mathrm{mL}$ to erythromycin. Data demonstrated that azithromycin can be a good choice of drug to treat patients infected by B. pertussis, in our country. The detailed data are listed in the Tables 1 and 2.

Following the PCR process, 23SrRNA PCR products were observed in all B. pertussis strains (Figure 4). After performing RFLP, results demonstrated that there was no cutting site for BbsI enzyme in the 23SrRNA PCR product of macrolide resistant B. pertussis (Figure 4). The result of sequencing showed that there was no cutting site for BbsI enzyme or transition mutation (A-G) at nucleotide positions 2047 or 2058 , in a critical region for erythromycin binding of the 23SrRNA gene.

\section{Discussion}

Due to poor lifelong immunity, whooping cough continues, despite national immunization programs. Widespread antibiotics were administered for the treatment and control of transition $B$. pertussis bacterium, leading to emergence of resistant strains. Then improvement of vaccines and vaccine schedules for pertussis is a necessity. Laboratory diagnosis of pertussis is traditionally based on culture, which is highly specific, even though it is maximally sensitive only in the initial phases of the disease (14).

There is a need for more rapid and sensitive diagnostic methods that have high positive predictive value, especially in the early stages of disease. As is the case for other fastidious organisms, PCR offers an attractive alternative for detecting $B$. pertussis and B. parapertussis in clinical specimens $(15,16)$. All positive $B$. pertussis colonies were confirmed by Taq Man RT-PCR. Erythromycin has been used in the USA for over 45 years, for the treatment of patients with pertussis and is currently recommended for prophylaxis, as well (16). The high level erythromycin resistant B. pertussis isolates in the USA carry an A-G transition at nucleotide position 2047 of the 23SrRNA gene, in a region critical for erythromycin binding (4).

We detected just one strain, as high level macrolide resistant B. pertussis. After performing RFLP-PCR and

\begin{tabular}{lcccccccc}
\hline \multicolumn{2}{l}{ Table 1. Detailed Data From Eleven Isolated Bacteria out of 1084 Specimens ${ }^{\text {a }}$} & & & \\
\hline Isolates No. & Strains & Age of Patients & Province of Iran & Year & PFGE Pattern & MIC (Eryth) & MIC (Clarith) & MIC (Azith) \\
\hline $\mathbf{1}$ & BpT1 & 2, mon & Tehran & 2009 & A1 & $<0.06$ & $<0.06$ & $<0.06$ \\
$\mathbf{2}$ & BpM1 & 3, mon & Mazandaran & 2009 & A1 & $<0.06$ & $<0.06$ & 0.125 \\
$\mathbf{3}$ & BpT2 & 3, mon & Tehran & 2009 & A1 & $<0.06$ & $<0.06$ & $<0.06$ \\
$\mathbf{4}$ & BpE1 & 2, y & Isfahan & 2009 & A2 & $<0.06$ & $<0.06$ & $<0.06$ \\
$\mathbf{5}$ & BpQ1 & $18, \mathrm{y}$ & Qom & 2009 & A3 & $<0.06$ & 2 & $<0.06$ \\
$\mathbf{6}$ & BpM2 & $3, \mathrm{y}$ & Mazandaran & 2009 & B & 4 & 8 & $<0.06$ \\
$\mathbf{7}$ & BpK1 & 5, mon & Khuzestan & 2010 & B & 4 & 32 & $<0.06$ \\
$\mathbf{8}$ & BpKO1 & $4, \mathrm{y}$ & Khorasan & 2009 & C & 4 & 8 & $<0.06$ \\
$\mathbf{9}$ & BpM3 & 1, mon & Mazandaran & 2009 & D & $<0.06$ & 0.5 & $<0.06$ \\
$\mathbf{1 0}$ & BpK2 & 1.5, mon & Khorasan & 2009 & E & 2 & $<0.06$ & $<0.06$ \\
$\mathbf{1 1}$ & BpK3 & $26, \mathrm{~d}$ & Khorasan & 2009 & F & 128 & $>256$ & $<0.06$ \\
\hline
\end{tabular}

a Abbreviations: Azith, azithromycin; Clarith, clarithromycin; d, days; Erythr, erythromycin; MIC, minimum inhibitory concentration; mon, months; y, years.

Table 2. Detailed Data From All Samples, Such as Age and Vaccinated Suspected Patients to Pertussis

\begin{tabular}{lcccc}
\hline Variables & \multicolumn{3}{c}{ Values } \\
\hline Specimens (Vaccinated) & 152 & $586(486)$ & $157(143)$ & $111(63)$ \\
Isolated Bacteria (Vaccinated) & $3(0)$ & $4(3)$ & $2(2)$ & $1(0)$
\end{tabular}


sequencing of 23SrRNA gene, in this strain, we realized that the high level resistance was not dependent on the A-G transition at nucleotide position 2047 or 2058 of the 23SrRNA gene. We suggest that other mechanisms, responsible for the resistance of this bacterium, should be considered. Given the low frequency of macrolides resistant B. pertussis, and to prevent the spread of these bacteria in high risk groups, a program for the identification of resistant bacteria in the laboratories and improvement of vaccines, vaccine schedules regarding pertussis, is necessary $(4-6,8)$.

\section{Acknowledgements}

Special thanks go to our dear colleges at the Medical Bacteriology Department Pasteur Institute of Iran, Tehran, Iran.

\section{Funding/Support}

This study was financially supported by Pasteur Institute of Iran, Tehran, Iran.

\section{References}

1. Bass JW. Erythromycin for treatment and prevention of pertussis. Pediatr Infect Dis. 1986;5(1):154-7.

2. Sandora TJ, Gidengil CA, Lee GM. Pertussis vaccination for health care workers. Clin Microbiol Rev. 2008;21(3):426-34.

3. Pertussis vaccines: WHO position paper. Wkly Epidemiol Rec. 2010;85(40):385-400.

4. Bartkus JM, Juni BA, Ehresmann K, Miller CA, Sanden GN, Cassiday PK, et al. Identification of a mutation associated with erythromycin resistance in Bordetella pertussis: implications for surveillance of antimicrobial resistance. J Clin Microbiol. 2003;41(3):1167-72.

5. Guillot S, Descours G, Gillet Y, Etienne J, Floret D, Guiso N. Macrolide-resistant Bordetella pertussis infection in newborn girl, France. Emerg Infect Dis. 2012;18(6):966-8.

6. Wang Z, Li Y, Hou T, Liu X, Liu Y, Yu T, et al. Appearance of macrolide-resistant Bordetella pertussis strains in China. Antimicrob Agents Chemother. 2013;57(10):5193-4.

7. Weisblum B. Erythromycin resistance by ribosome modification. Antimicrob Agents Chemother. 1995;39(3):577-85.

8. Hill BC, Baker CN, Tenover FC. A simplified method for testing Bordetella pertussis for resistance to erythromycin and other antimicrobial agents. J Clin Microbiol. 2000;38(3):1151-5.

9. WHO. Laboratory manual for the diagnosis of whooping cough caused by Bordetella pertussis/Bordetella parapertussis. 2007.

10. Reischl U, Lehn N, Sanden GN, Loeffelholz MJ. Real-time PCR assay targeting IS481 of Bordetella pertussis and molecular basis for detecting Bordetella holmesii. J Clin Microbiol. 2001;39(5):1963-6.

11. Probert WS, Ely J, Schrader K, Atwell J, Nossoff A, Kwan S. Identification and evaluation of new target sequences for specific detection of Bordetella pertussis by real-time PCR. J Clin Microbiol. 2008;46(10):3228-31.

12. Jorgensen JH, Clinical Laboratory Standards Institute. Methods for Antimicrobial Dilution and Disk Susceptibility Testing of Infrequently Isolated or Fastidious Bacteria: Approved Guideline. National Committee for Clinical Laboratory Standards; 2010.

13. Advani A, Donnelly D, Hallander H. Reference system for characterization of Bordetella pertussis pulsed-field gel electrophoresis profiles. J Clin Microbiol. 2004;42(7):2890-7.

14. Loeffelholz MJ, Thompson CJ, Long KS, Gilchrist MJ. Detection of Bordetella holmesii using Bordetella pertussis IS481 PCR assay. J Clin Microbiol. 2000;38(1):467.

15. Backman A, Johansson B, Olcen P. Nested PCR optimized for detection of Bordetella pertussis in clinical nasopharyngeal samples. J Clin Microbiol. 1994;32(10):2544-8.

16. Farrell DJ, McKeon M, Daggard G, Loeffelholz MJ, Thompson CJ, Mukkur TK. Rapid-cycle PCR method to detect Bordetella pertussis that fulfills all consensus recommendations for use of PCR in diagnosis of pertussis. J Clin Microbiol. 2000;38(12):4499-502. 